\title{
Synchronous Bilateral Carcinoma of the Ureters. Report of a Case and a Short Discussion of the Literature
}

\author{
DIMITRIOS SIDIROPOULOS ${ }^{1,2^{*}}$, FILIPPOS KAPOGIANNIS ${ }^{3 *}$, PANAGIOTA KRIPOURI $^{2}$, \\ DIMITRIOS FILIPPOU ${ }^{2}$ and KONSTANTINOS VLASSIS ${ }^{2}$ \\ ${ }^{1}$ Department of Urology, General Hospital of Katerini, Katerini, Greece; \\ ${ }^{2}$ Department of Anatomy and Surgical Anatomy, Medical School, \\ National and Kapodistrian University of Athens, Athens, Greece; \\ ${ }^{3}$ Department of Urology, Hippokrateion General Hospital of Athens, Athens, Greece
}

\begin{abstract}
Background/Aim: The strategy for treatment of bilateral simultaneous renal pelvic and/or ureteral tumors has not been established and is dependent on individual decision. Case Report: We herein report the case of an elderly man who was examined for fatigue at the emergency department and subsequently diagnosed with bilateral ureteral cancer. The most common histologic type of ureteral cancer is urothelial (transitional epithelium) cancer and though it is rare, it can be synchronously present in the contralateral ureter. Computed tomography-urography (CTU) has very good results regarding diagnosis and surgical treatment and -though not preferred in our case-, it has shown promising results. Conclusion: We believe that conservative treatment is a viable therapeutic option for simultaneous bilateral renal pelvic and/or ureteral tumors in selected patients. Yet, this treatment has seldom been performed, although standard therapy with bilateral nephroureterectomy inevitably requires the support of hemodialysis or renal transplantation.
\end{abstract}

Synchronous lesions of the ureters are those found in the same radiological study, while metachronous lesions are found in future exams (1). We hereby discuss the case of an elderly man who was diagnosed with synchronous lesions of both ureters with only fatigue as an initial symptom. The

This article is freely accessible online.

*These Authors contributed equally to this study and should be regarded as first Authors.

Correspondence to: Dimitrios Sidiropoulos, Department of Urology, General Hospital of Katerini, 7th Merarhias 26, Katerini 60100, Greece. Tel: +30 2351350200, e-mail: sidiropd2@hotmail.com

Key Words: Bilateral cancer, ureter, synchronous, comorbidities. patient received palliative care due to reluctance to undergo therapy and multiple comorbidities. Upper urinary tract urothelial carcinomas (UTUC) are distinct clinical entities and differ in histology and prognosis; however, the majority can cause hematuria and if they are grouped as high-risk, they have the tendency to progress and ultimately shorten the overall survival, which should be kept in the mind of the general practitioner so as to urgently refer the case to an urologist (2).

\section{Case Report}

A 79-year-old man was investigated in our hospital for tiredness. Upon history examination, he revealed that he experienced gross hematuria without any pain for the last three months. He was also aware that he was anemic at least for a year after his annual check-up but the cause of anemia was never sought. His medical history revealed hypercholesterolemia, benign prostatic hyperplasia, stage III chronic obstructive pulmonary disease, heart failure, atrial fibrillation and chronic angina. He also was a heavy smoker for two decades and had quitted two months ago. On admission he had a hematocrit of $26 \%$. Diagnostic cytology showed atypical urothelial cells. He was subjected to flexible cystoscopy which revealed a solitary, small, non-hemorrhagic papillary lesion on the posterior wall of the bladder (Figure 1). During the observation of the ureteral orifices, the right urine jet was remarkably bloody. After cystoscopy the patient underwent a CT exam of the thorax, abdomen and the retroperitoneal space (CTU with delayed images) where both ureters were found to have solid lesions (Figures 2-4).

The patient and his closest family were thoroughly informed about the findings and asked for their consent for surgical management, albeit they refused to give it. The surgical plan was to perform a transurethral resection of the exophytic lesion of the bladder wall, followed by kidneysparing surgery or bilateral radical nephroureterectomy 

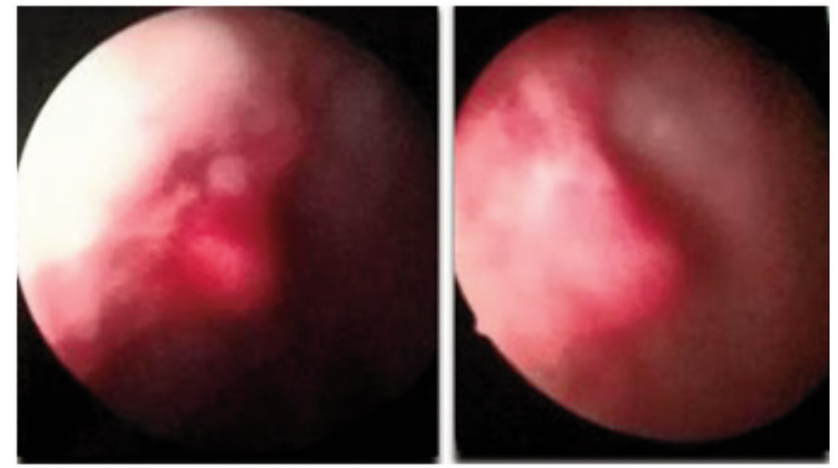

Figure 1. Cystoscopic images - lesion of the posterior wall of the bladder.

(RNU) and creation of an arteriovenous fistula for a vascular access for long-term hemodialysis. Due to his age, his previous cardiopulmonary poor state and given his understandable reluctance to undergo major surgery, palliative care was preferred. The patient was prescribed analgesics and was advised to undergo frequent blood tests to sustain an acceptable hematocrit level around $30 \%$. The patient has remained without radiologic evidence of disease progression after one year of follow-up. He has retained his performance status (ECOG-0, CCI-3), eGFR level= $84 \mathrm{ml} / \mathrm{min}$ and life quality (according to SF-36). His only bothering symptom is intermittent mild macroscopic hematuria without need for catheterization so far. We thus assume that despite the absence of histology specimen and the vigorous but short follow-up period, our patient harbors low-risk disease.

\section{Discussion}

Synchronous bilateral transitional cell UTUCs are found in $2 \%$ to $4 \%$ of all cases of transitional cell carcinomas and 20 to $50 \%$ of UTUC cases are estimated to have urinary bladder involvement sometime in their life $(3,4)$. CTU is a standard method in detecting ureter malignancies (5), since it has shown excellent diagnostic results with both high specificity and sensitivity as discussed by Froemming et al. (6). Squamous cell carcinoma and urothelial cancer are not distinguishable in CTU (1), however we believe that given its bilateral presentation and the synchronous involvement of the bladder, the likelihood of any other type of cancer would be minimal in this case.

Known risks factors for developing urothelial carcinomas include the use of tobacco, (smokers having an up to sevenfold greater risk) $(3,7)$ previous bladder or kidney cancer and gender, with men being more susceptible than women, especially at an old age $(5,7)$.

Currently, no biomarker is routinely used for this type of cancer, which is unfortunate, because this specific type is

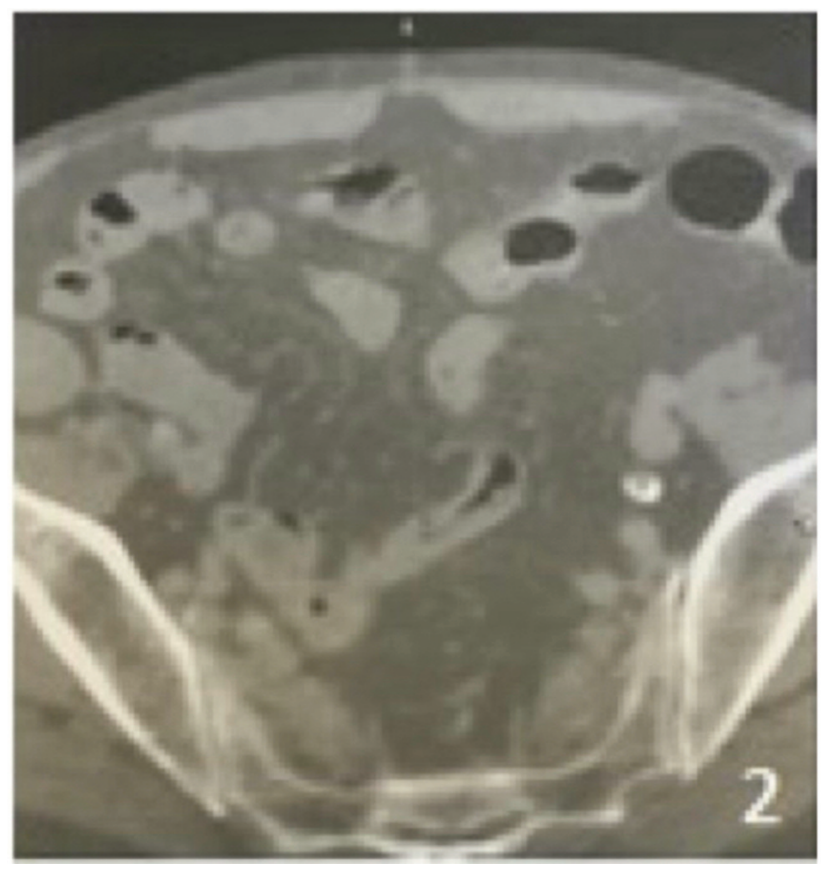

Figure 2. An axial computed tomography (CT) image obtained during the excretory phase shows small filling defect of the left ureter.

usually asymptomatic until it has progressed. Even though urine cytology has been proven as a helpful supplementary method in diagnosing upper tract malignancies, it is still early before it is fully intergraded with reliable biomarkers to boost its sensitivity (8). In the last years, several studies have focused in discovering the ideal markers for early detection and/or better screening and follow up of patients. FDA has approved some markers, which are currently commercially available, albeit research is still needed before they substitute the standard follow-up method of cystoscopy or cytology (9). Molecular classification by evaluating DNA, RNA and protein expression has identified four molecular subtypes with distinct clinical behaviours, but, as yet, it is unclear whether these subtypes will respond differently to treatment (10).

As for the surgical treatment, kidney-sparing surgery for low-risk UTUC reduces the morbidity associated with radical surgery (e.g. loss of kidney function), without compromising oncological outcomes and represents the preferred approach as survival is similar to that after RNU (11). This option should therefore be discussed in all low-risk cases, irrespective of the status of the contralateral kidney. Contemplation of anything less than total excision must take into account the potential risk for tumor recurrence anywhere in the upper tract unit. In other than unifocal, low-grade, low-stage renal pelvic tumors, the probable extensive involvement of both contiguous and noncontiguous sites would appear to make segmental excision an unnecessary option with a potentially serious risk. Open RNU with bladder cuff excision, performed 


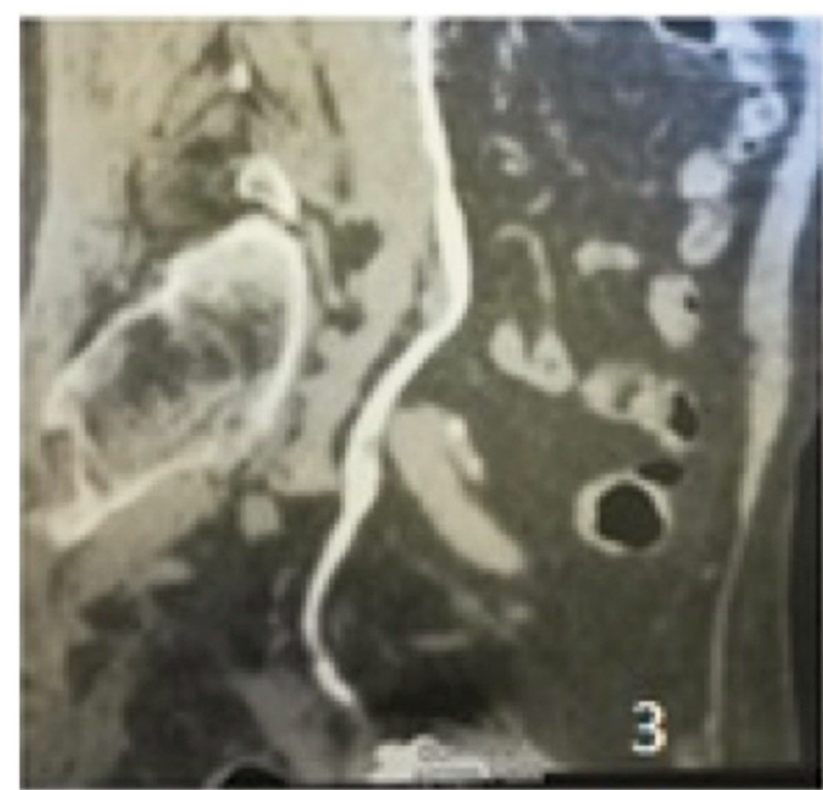

Figure 3. Coronal computed tomography urography (CTU) image shows mild wall thickening in the middle and filling defects in the lower third of the right ureter.

according to oncological principles preventing tumour seeding, is the standard treatment of high-risk UTUC, regardless of tumour location (12). Oncological outcomes after RNU have not changed significantly over the past three decades despite staging and surgical refinements (13).

The low incident of bilateral disease has shown that the clinical cases differ by their features and often need individual strategy development as data about the correct management are scarce and controversial. The long-term outcome for patients with synchronous tumors is largely unknown, because our current knowledge is based primarily on approximately 40 case reports with short follow-up. Futhermore, survival rates of ureteral carcinomas cohort suggest a need for further search of the ways to improve treatment results.

\section{Conclusion}

Urothelial cancer is a complex disease that is often followed by chronic kidney failure prior or after surgery and requires a high index of clinical suspicion to be diagnosed, since it rarely causes bothering symptoms and has a relative high mortality. Bilateral ureteral carcinomas are rare and usually have poor prognosis even if treated with RNU, unless histology shows low grade disease and a more conservative therapy is advocated. The kidney preserving strategy can be considered as an option not only in low-, but also in highrisk patients. Under certain conditions however, palliative and/or supportive care can provide an adequate health-related

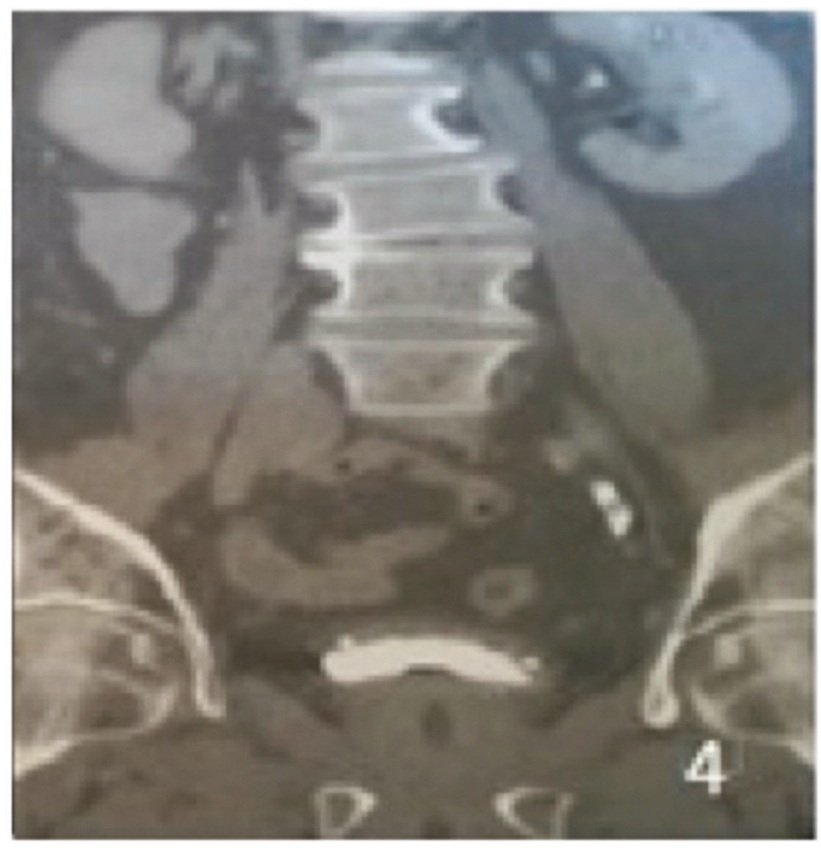

Figure 4. Coronal computed tomography urography (CTU) image shows abnormal filling defect in the left ureter compatible with urothelial cancer.

quality of life and should not always be undertaken as a last resort. In our mind, in such cases, renal function is crucial and remains one of the main parameters that can influence outcomes. This patient has remained without evidence of disease progression for one year. This article focused on a case of a rare bilateral carcinoma of the ureters aiming to briefly present the diagnosis and treatment plans of such a malignancy and the hopes and goals of urological society for treating upper tract cancers in the future.

\section{Conflicts of Interest}

The Authors declare that there are no conflicts of interest regarding the publication of this paper.

\section{Authors' Contributions}

DS and FK reviewed literature data; KP, DF prepared the draft of the manuscript; SD and VK were advisors of the therapy procedures. DS reviewed the final version of the manuscript. All Authors read and approved the final version of the manuscript.

\section{References}

1 Potenta SE, D'Agostino R, Sternberg KM, Tatsumi K and Perusse K: CT urography for evaluation of the ureter. Radiographics 35(3): 709-726, 2015. PMID: 25815907. DOI: 10.1148/rg.2015140209 
2 Arora HC, Fascelli M, Zhang JH, Isharwal S and Campbell SC: Kidney, ureteral, and bladder cancer: A primer for the internist. Med Clin North Am 102(2): 231-249, 2018. PMID: 29406055. DOI: $10.1016 /$ j.mcna.2017.10.002

3 Kirkali Z and Tuzel E: Transitional cell carcinoma of the ureter and renal pelvis. Crit Rev Oncol Hematol 47(2): 155-169, 2003. DOI: $10.1016 / \mathrm{s} 1040-8428(03) 00079-9$

4 Buldu I, Kurt O, Inan R and Istanbulluoglu O: A case of synchronous bilateral upper urinary system urothelial carcinoma. J Clin Anal Med 7(2): 265-267, 2016. DOI: 10.4328/JCAM.4172

5 Roupret M, Babjuk M, Comperat E, Zigeuner R, Sylvester RJ, Burger M, Cowan NC, Bohle A, Van Rhijn BW, Kaasinen E, Palou J and Shariat SF: European association of urology guidelines on upper urinary tract urothelial cell carcinoma: 2015 update. Eur Urol 68(5): 868-879, 2015. PMID: 26188393. DOI: 10.1016/j.eururo.2015.06.044

6 Froemming A, Potretzke T, Takahashi N and Kim B: Upper tract urothelial cancer. Eur J Radiol 98: 50-60, 2018. PMID: 29279170. DOI: 10.1016/j.ejrad.2017.10.021

7 Miyazaki J and Nishiyama H: Epidemiology of urothelial carcinoma. Int J Urol 24(10): 730-734, 2017. PMID: 28543959. DOI: $10.1111 /$ iju. 13376

8 Xing J and Reynolds JP: Diagnostic advances in urine cytology. Surg Pathol Clin 11(3): 601-610, 2018. PMID: 30190143. DOI: 10.1016/j.path.2018.06.001

9 Tabayoyong W and Kamat AM: Current use and promise of urinary markers for urothelial cancer. Curr Urol Rep 19(12): 96, 2018. PMID: 30328534. DOI: 10.1007/s11934-018-0857-1

10 Moss T.J, Qi Y, Xi L, Peng B, Kim TB, Ezzedine NE, Mosqueda ME, Guo CC, Czerniak BA, Ittmann M, Wheeler DA, Lerner SP and Matin SF: Comprehensive genomic characterization of upper tract urothelial carcinoma. Eur Urol 72(4): 641-649, 2017. PMID: 28601352. DOI: 10.1016/j.eururo.2017.05.048
11 Seisen T, Peyronnet B, Dominguez-Escrig JL, Bruins HM, Yuan CY, Babjuk M, Böhle A, Burger M, Compérat EM, Cowan NC, Kaasinen E, Palou J, van Rhijn BW, Sylvester RJ, Zigeuner R, Shariat SF and Rouprêt M: oncologic outcomes of kidney-sparing surgery versus radical nephroureterectomy for upper tract urothelial carcinoma: A systematic review by the EAU non-muscle invasive bladder cancer guidelines panel. Eur Urol 70(6): 1052-1068, 2016. PMID: 27477528. DOI: 10.1016/j.eururo.2016.07.01

12 Margulis V, Shariat SF, Matin SF, Kamat AM, Zigeuner R, Kikuchi E, Lotan Y, Weizer A, Raman JD, Wood CG and The Upper Tract Urothelial Carcinoma Collaboration: Outcomes of radical nephroureterectomy: a series from the Upper Tract Urothelial Carcinoma Collaboration. Cancer 115(6): 1224-1233, 2009. PMID: 19156917. DOI:10.1002/cncr.24135

13 Adibi M, Youssef R, Shariat SF, Lotan Y, Wood CG, Sagalowsky AI, Zigeuner R, Montorsi F, Bolenz C and Margulis V: Oncological outcomes after radical nephroureterectomy for upper tract urothelial carcinoma: comparison over the three decades. Int J Urol 19(12): 1060-1066, 2012. PMID: 22882743. DOI: $10.1111 / \mathrm{j} .1442-2042.2012 .03110 . \mathrm{x}$

Received March 15, 2020

Revised March 25, 2020

Accepted March 27, 2020 